\title{
On the class of multivalent analytic functions defined by differential operator for derivative of first order
}

\author{
Zainab Odeh A.Mohammed \\ Department of Mathematics \\ College of Education \\ University of AL-Qadisiyah \\ AL-Qadisiya -Iraq \\ hsyn47421@gmail.com
}

\author{
Kassim A. Jassim \\ Department of Mathematics \\ College of Science \\ University of Baghdad \\ Baghdad-Iraq \\ Kasimmathphd@gmail.com
}

Available online : $\quad 28 / 1 / 2019$

DOI: $10.29304 / j q c m .2019 .11 .1 .475$

\begin{abstract}
In the submitted search ,by making use of Differential operator, we drive coefficient bounds and some important properties of the subclass $T_{j}(n, p, q, \alpha, \lambda) \quad(p, j \in N=$ $\{1,2, \ldots\} ; q, n \in N_{0}=N \cup\{0\} ; 0 \leq \alpha<p-q$ ) of analytic

and multivalent function with negative coefficients .Distortion property for functions in the class $T_{j}(n, p, q, \alpha, \lambda)$ are investigated once by using the composition involving an integral operator and certain fractional calculus operator and other once by using the composition involving an integral operator and certain fractional calculus inverse operator .
\end{abstract}

Keywords. Multivalent function, Coefficient bounds ,Distortion inequality, $\delta$-neighbourhood, Differential operator, integral and fractional operators .

Mathematics Subject Classification: 30C45. 
Zainab .O/ Kassim .A

\section{1-Introduction}

Let $T(j, p)$ be the class of analytic and multivalent functions $f(z)$ in the open unit disk

$U=\{z: z \in C:|z|<1\}$ that defined by

$$
f(z)=z^{p}-\sum_{k=j+p}^{\infty} a_{k} z^{k} \quad\left(a_{k} \geq\right.
$$

$0 ; j, p \in N=\{1,2, \ldots$.$\} .$

Let $T(j, p)$ the class consists of function of the form

$f^{q}(z)=\frac{p !}{(p-q) !} z^{p-q}-\sum_{k=j+p}^{\infty} \frac{k !}{(k-q) !} a_{k} z^{k-q}$

$\left(a_{k} \geq 0 ; q \in N_{0}=N \cup\{0\} ; p>q\right)$

(2)

The Differential operator for a function in $T(j, p)$ is define by

$$
\begin{aligned}
& D_{p}^{n}\left(f^{q}(z)\right)= \\
& \frac{p !}{(p-q) !} z^{p-q}-\sum_{k=j+p}^{\infty} \frac{k !}{(k-q) !}\left(\frac{k-q}{p-q}\right)^{n} a_{k} z^{k-q} \\
& \left(p, j \in N=\{1,2, \ldots\} ; q, n \in N_{0} ; p>q\right)
\end{aligned}
$$

(3)

The operator $D_{p}^{n}$ was studied by M.K. Aouf [5] and Altintas et al. [7] , earlier by Owa [13], Yamakawa[9] ,Owa [12] ,Srivastava Owa[4] . It is easy to see that

$$
D_{p}^{n+1} f^{q}(z)=\frac{z}{(p-q)}\left(D_{p}^{n} f^{q}(z)\right)^{\prime}
$$

By using the operator $D_{p}^{n} f^{q}(z)$ Given by (3) ,a function $f(z)$ belonging to $T_{j}(n, p, q, \alpha, \lambda)$ if and only if

$R\left(\frac{D_{p}^{n+1}\left(f^{q}(z)\right)}{(1-\lambda) D_{p}^{n}\left(f^{q}(z)\right)+\lambda D_{p}^{n+1}\left(f^{q}(z)\right)}\right)>\alpha(p \in N ; q, n \in$ $\left.N_{0}=N \cup\{0\} ; p>q\right)$, (5)

for some $\alpha(0 \leq \alpha<p)$ and for all $z \in U$.

Next the following earlier investigations by Osman Altintas,Huseyin Irmak and H.M.Srivastava [6] ,

when $f(z) \in T(j, p)$ we define the $\delta$ - neighborhood by

$$
N_{\delta}(f)=\left\{g: g \in T(j, p), g(z)=z^{p}-\right.
$$

$\sum_{k=j+p}^{\infty} b_{k} z^{k}$

and $\left.\sum_{k=j+p}^{\infty} k\left|a_{k}-b_{k}\right| \leq \delta\right\}$.

(6)

So that ,obviously ,

$N_{\delta}(h)=\left\{g: g \in T(j, p), g(z)=z^{p}-\right.$

$\sum_{k=j+p}^{\infty} b_{k} z^{k}$

And $\left.\sum_{k=j+p}^{\infty}\left|b_{k}\right| \leq \delta\right\} \quad$ (7)

Where, and in what follows,

$$
h(z)=z^{p} \quad\left(k \geq j+p ; n, p \in N ; q \in N_{0}=\right.
$$

$N \cup\{0\})$.

we using the familiar operator $J_{c, p}$ defined by Bernardi [10], Libera[8] and Srivastave et al. [2] as follows

$\begin{array}{ll}\left(J_{c, p} f\right)(z)=\frac{c+p}{z^{c}} \int_{0}^{z} t^{c-1} f(t) d t & (f(z) \in \\ T(j, p) ; c>-p ; p \in N), & \text { (9) }\end{array}$ and fractional calculus operator $D_{z}^{\mu}$ Srivastave [11] , Srivastava et al.[3] that known as the form

$D_{z}^{\mu}\left(z^{p}\right)=\frac{\Gamma(p+1)}{\Gamma(p-\mu+1)} z^{p-\mu} \quad(p>-1 ; \mu \in R)$

\section{2-Coefficient Inequalities}

We drive sufficient condition for $f(z)$ that defined by using differential operator .

Theorem 1. Assume that $f(z) \in T(j, p)$. Then $f(z) \in T_{j}(n, p, q, \alpha, \lambda)$ if and only if

$$
\begin{aligned}
& \sum_{k=j+p}^{\infty}\left(\frac{k-q}{p-q}\right)^{n}\left[\left(\frac{k-q}{p-q}\right)-\alpha\left(1+\lambda\left(\frac{k-q}{p-q}-\right.\right.\right. \\
& \text { 1)) }] \delta(k, q) a_{k}<(1-\alpha) \delta(p, q) \\
& \left(0 \leq \alpha<p-q ; p, j \in N ; q, n \in N_{0} ; p>q\right)
\end{aligned}
$$$$
\text { (11) }
$$

Where

$\delta(p, q)=\frac{p !}{(p-q) !}= \begin{cases}p(p-1) \ldots \ldots(p-q+1) & q \neq 0 \\ 1 & q=0\end{cases}$

Proof. If $f(z) \in T_{j}(n, p, q, \alpha, \lambda)$, then

$R\left(\frac{D_{p}^{n+1}\left(f^{q}(z)\right)}{(1-\lambda) D_{p}^{n}\left(f^{q}(z)\right)+\lambda D_{p}^{n+1}\left(f^{q}(z)\right)}\right)=$ $R\left(\frac{\frac{p !}{(p-q) !}-\sum_{k=j+p}^{\infty} \frac{k !}{(k-q) !}\left(\frac{k-q}{p-q}\right)^{n+1} a_{k} z^{k-p}}{\left.\frac{p !}{(p-q) !}-\sum_{k=j+p(k-q) !}^{\infty} \frac{k !}{p-q}\right)^{n}\left[1-\lambda+\lambda\left(\frac{k-q}{p-q}\right)\right] a_{k} z^{k-p}}\right)$

$\left\{\frac{\frac{p !}{(p-q) !} \sum_{k=j+p}^{\infty} \frac{k !}{(k-q) !}\left(\frac{k-q}{p-q}\right)^{n+1} a_{k} z^{k-p}}{\frac{p !}{(p-q) !}+\sum_{k=j+p}^{\infty} \frac{k !}{(k-q) !}\left(\frac{k-q}{p-q}\right)^{n}\left[\lambda-1-\lambda\left(\frac{k-q}{p-q}\right)\right] a_{k} z^{k-p}}\right\}>\alpha$

$(1-\alpha) \frac{p !}{(p-q) !}>\sum_{k=j+p}^{\infty}\left(\frac{k-q}{p-q}\right)^{n}\left(\left(\frac{k-q}{p-q}\right)+\lambda \alpha-\alpha-\right.$ $\left.\lambda \alpha\left(\frac{k-q}{p-q}\right)\right) \frac{k !}{(k-q) !} a_{k} z^{k-p}$

Since $\quad \mathrm{z} \rightarrow 1^{-}$,we have $\sum_{k=j+p}^{\infty}\left(\frac{k-q}{p-q}\right)^{n}\left(\left(\frac{k-q}{p-q}\right)-\right.$ $\left.\alpha\left(1+\lambda\left(\left(\frac{k-q}{p-q}\right)-1\right)\right)\right) \delta(k, q) a_{k} z^{k-p}<(1-$

$\alpha) \delta(p, q)$

Conversely ,assume that inequality (11) holds true , since

$R(w)>\alpha$ if and only if $\left|\frac{w-1}{w+(1-2 \alpha)}\right|<1$

Since

$$
\left|\frac{\frac{D_{p}^{n+1}\left(f^{q}(z)\right)}{(1-\lambda) D_{p}^{n}\left(f^{q}(z)\right)+\lambda D_{p}^{n+1}\left(f^{q}(z)\right)}-1}{\frac{D_{p}^{n+1}\left(f^{q}(z)\right)}{(1-\lambda) D_{p}^{n}\left(f^{q}(z)\right)+\lambda D_{p}^{n+1}\left(f^{q}(z)\right)}+(1-2 \alpha)}\right|
$$


$=\left|\frac{(1-\lambda) D_{p}^{n+1}\left(f^{q}(z)\right)-(1-\lambda) D_{p}^{n}\left(f^{q}(z)\right)}{(1+\lambda-2 \alpha \lambda) D_{p}^{n+1}\left(f^{q}(z)\right)+(1-2 \alpha)-\lambda(1-2 \alpha) D_{p}^{n}\left(f^{q}(z)\right)}\right|$

$=\frac{-\sum_{k=j+p}^{\infty} \frac{k !}{(k-q) !}\left(\frac{k-q}{p-q}\right)^{n}\left[\frac{k-q}{p-q}-\lambda\left(\frac{k-q}{p-q}\right)-1+\lambda\right] a_{k} z^{k-p}}{2 \frac{p !}{(p-q) !}(1-\alpha)+\sum_{k=j+p}^{\infty} \frac{k !}{(k-q) !}\left(\frac{k-q}{p-q}\right)^{n}\left[2 \alpha+\lambda-2 \alpha \lambda-1-\frac{k-q}{p-q}-\lambda\left(\frac{k-q}{p-q}\right)+2 \alpha \lambda\left(\frac{k-q}{p-q}\right)\right] a_{k} z^{k-p}} \mid$

$\leq \frac{\sum_{k=j+p}^{\infty}\left(\frac{k-q}{p-q}\right)^{n}\left[\left(\frac{k-q}{p-q}\right)-\lambda\left(\frac{k-q}{p-q}\right)-1+\lambda\right] \frac{k !}{(k-q) !} a_{k} z^{k-p}}{\left.2(1-\alpha) \frac{p !}{(p-q) !}+\sum_{k=j+p}^{\infty} \frac{k-q}{p-q}\right)^{n}\left[2 \alpha+\lambda-2 \alpha \lambda-1-\left(\frac{k-q}{p-q}\right)\{1+\lambda-2 \alpha \lambda\}\right] \frac{k !}{(k-q) !} a_{k} z^{k-p}}$

$$
\leq 1
$$

Putting $j=1, n=1, q=0$ and $\lambda=0$ in Theorem 1 ,we have the following corollary :

Corollary 1 . Let the function $f(z) \in T(j, p)$. Then $f(z) \in C(p, \alpha)$ if and only if

$\sum_{k=1+p}^{\infty} \frac{k}{p}\left[\frac{k}{p}-\alpha\right] a_{k}<(1-\alpha)$

$(0 \leq \alpha<$ $p ; p \in N)$.

Not that this result obtained by Salagean et al[1] .

Corollary 2. Assume that the function $f(z)$ defined by (2) be in the class $T_{j}(n, p, q, \alpha, \lambda)$.Then

$$
\sum_{k=j+p}^{\infty} \delta(k, q) a_{k}<\frac{(1-\alpha) \delta(p, q)}{\left(\frac{j}{p-q}+1\right)^{n}\left(\left(\frac{j}{p-q}(1-\alpha \lambda)\right)+(1-\alpha)\right)}
$$

$\left(k \geq j+p ; p, j \in N ; q, n \in N_{0} ; p>q\right)$.

The result is sharp for the function $f(z)$ given by

$$
f(z)=
$$$$
z^{p}-
$$$$
\left(\frac{(1-\alpha) \delta(p, q)}{\left(\frac{k-q}{p-q}\right)^{n}\left(\left(\frac{k-q}{p-q}\right)-\alpha\left(1+\lambda\left(\left(\frac{k-q}{p-q}\right)-1\right)\right)\right) \delta(k, q)}\right) z^{k} \quad(k \geq j+
$$

$\left.p ; p, j \in N ; q, n \in N_{0} ; p>q\right)$.

\section{3-Extreme points}

Theorem 2. Let $f_{p}(z)=z^{p}$ and $f_{k}(z)=z^{p}-$ $\left(\frac{(1-\alpha) \delta(p, q)}{\left(\frac{k-q}{p-q}\right)^{n}\left(\left(\frac{k-q}{p-q}\right)-\alpha\left(1+\lambda\left(\left(\frac{k-q}{p-q}\right)-1\right)\right)\right) \delta(k, q)}\right) z^{k}$,

for $k \geq j+p$ and $p>q$. Then $f(z) \in$ $T_{j}(n, p, q, \alpha, \lambda)$ if and only if it is of the form

$f(z)=\sum_{k=p}^{\infty} \omega_{k} f_{k}(z)$,where $\omega_{k} \geq 0$ for all $k \geq j+p$ and $\sum_{k=p}^{\infty} \omega_{k}=1$.

Proof . Suppose that $\quad f(z)=\sum_{k=p}^{\infty} \omega_{k} f_{k}(z)=$ $\omega_{p} f_{p}(z)+\sum_{k=j+p}^{\infty} \omega_{k} f_{k}(z)$

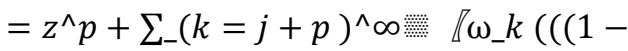

$\alpha) \delta(p, q)) /\left(((k-q) /(p-q))^{\wedge} n(((k-q) /(p-\right.$

$q))-\alpha(1+\lambda(((k-q) /(p-q))-$

1))) $\delta(k, q))) z^{\wedge} k \rrbracket$.
Then

$$
\sum_{k=j+p}^{\infty}\left(\frac{k-q}{p-q}\right)^{n}\left(\left(\frac{k-q}{p-q}\right)-\alpha(1+\right.
$$

$\lambda\left(\left(\frac{k-q}{p-q}\right)-\right.$

1)) $\delta(k, q) \omega_{k}\left[\frac{(1-\alpha) \delta(p, q)}{\left(\frac{k-q}{p-q}\right)^{n}\left(\left(\frac{k-q}{p-q}\right)-\alpha\left(1+\lambda\left(\left(\frac{k-q}{p-q}\right)-1\right)\right)\right) \delta(k, q)}\right]$

$=\sum_{k=j+p}^{\infty} \omega_{k}(1-\alpha) \delta(p, q)$

$=\left(1-\omega_{p}\right)(1-\alpha) \delta(p, q)$

$\leq(1-\alpha) \delta(p, q)$.

Thus ,it follows from Theorem (1) that $f(z) \in$ $T_{j}(n, p, q, \alpha, \lambda)$.

Conversely ,suppose that $f(z) \quad \in$

$T_{j}(n, p, q, \alpha, \lambda)$, since

$a_{k}<\frac{(1-\alpha) \delta(p, q)}{\left(\frac{j}{p-q}+1\right)^{n}\left[\frac{j}{p-q}(1-\alpha \lambda)+(1-\alpha)\right] \delta(j+p, q)}, k \geq j+p$.

We define

$\omega_{k}=\frac{\left(\frac{k-q}{p-q}\right)^{n}\left(\left(\frac{k-q}{p-q}\right)-\alpha\left(1+\lambda\left(\left(\frac{k-q}{p-q}\right)-1\right)\right)\right) \delta(k, q)}{(1-\alpha) \delta(p, q)} a_{k}$

$(k \geq j+p)$.

And $\quad \omega_{p}=1-\sum_{k=j+p}^{\infty} \omega_{k} \quad$ by simple calculation, we get

$f(z)=z^{p}-\sum_{k=j+p}^{\infty} a_{k} z^{k}$

$=z^{p}$

$-\sum_{k=j+p}^{\infty}\left(\frac{(1-\alpha) \delta(p, q)}{\left(\frac{k-q}{p-q}\right)^{n}\left(\left(\frac{k-q}{p-q}\right)-\alpha\left(1+\lambda\left(\left(\frac{k-q}{p-q}\right)-1\right)\right)\right) \delta(k, q)}\right) \omega_{k} z^{k}$

$\sum_{k=j+p}^{\infty} \omega_{k} f(z)$

Thus we get the result .

4-Neighbouhoods for the function class $\boldsymbol{T}_{j}(\boldsymbol{n}, \boldsymbol{p}, \boldsymbol{q}, \alpha, \lambda)$

In this section, we conclude the neighborhood properties for each of the following slightly mutated function in the class $T_{j}(n, p, q, \alpha, \lambda, \gamma)$.Our first implication relation including the $\delta$ - neighbourhood $N_{\delta}(h)$ is given below in the following Theorem .

Theorem 3. If $f(z)$ belonging to $T_{j}(n, p, q, \alpha, \lambda)$,then

$T_{j}(n, p, q, \alpha, \lambda) \subset N_{\delta}(h)$.

Where $h(z)$ is defined as (8) and

$\delta:=\frac{(1-\alpha) \delta(p, q)}{\left(\frac{j}{p-q}+1\right)^{n}\left(\left(\frac{j}{p-q}(1-\alpha \lambda)\right)+(1-\alpha)\right)}$ 
Zainab .O/ Kassim .A

Proof - For $f(z) \in T(j, p) \quad$,Theorem

(1), immediately yields .

$$
\left(\frac{j}{p-q}+1\right)^{n}\left(\left(\frac{j}{p-q}(1-\alpha \lambda)\right)+(1-\alpha)\right) \delta(j+
$$

$p, q) \sum_{k=j+p}^{\infty} a_{k} \leq(1-\alpha) \delta(p, q)$,

$$
\left(\frac{\delta(j+p, q) \sum_{k=j+p}^{\infty} k a_{k} \leq}{\left(\frac{j}{p-q}+1\right)^{n}\left(\left(\frac{j}{p-q}(1-\alpha \lambda)\right)+(1-\alpha)\right)}\right) .
$$

Thus, we have

$$
\frac{\sum_{k=j+p}^{\infty} k a_{k} \leq}{\left(\frac{j}{p-q}+1\right)^{n}\left(\left(\frac{j}{p-q}(1-\alpha \lambda)\right)+(1-\alpha)\right) \delta(j+p, q)}:=\delta .
$$

This complete the proof .

A function $f(z) \in T(j, p)$ is said to be in the class $T_{j}(n, p, q, \alpha, \lambda, \gamma)$ if there exists another function $g(z) \in T_{j}(n, p, q, \alpha, \lambda, \gamma)$ such that

$$
\left|\frac{f(z)}{g(z)}-1\right|<p-\gamma \quad(z \in U: 0 \leq \gamma<p) \text {. }
$$

Theorem 4. Let $g(z) \in T_{j}(n, p, q, \alpha, \lambda, \gamma)$.Suppose also that

$$
\begin{aligned}
& \gamma= \\
& p- \\
& \frac{\delta}{j+p}\left[\frac{(j+p) !\left(\frac{j}{p-q}+1\right)^{n}\left(\left(\frac{j}{p-q}(1-\alpha \lambda)\right)+(1-\alpha)\right)}{\left(\frac{j}{p-q}+1\right)^{n}\left(\left(\frac{j}{p-q}(1-\alpha \lambda)\right)+(1-\alpha)\right)(j+p) !-(1-\alpha) \delta(p, q)(j+p-q) !}\right]
\end{aligned}
$$$$
\text { (16) }
$$

Then

$$
N_{\delta}(g) \subset T_{j}(n, p, q, \alpha, \lambda, \gamma)
$$

Proof . Suppose that $f(z) \in N_{\delta}(g)$, we then find from (6) that

$$
\sum_{k=j+p}^{\infty} k\left|a_{k}-b_{k}\right| \leq \delta
$$

Which readily implies the following coefficient inequality

$$
\sum_{k=j+p}^{\infty}\left|a_{k}-b_{k}\right| \leq \frac{\delta}{(j+p)} \quad,(j, p \in N ; p>q)
$$

Next ,since $g(z) \in T_{j}(n, p, q, \alpha, \lambda)$, we have

$$
\sum_{k=j+p}^{\infty} b_{k} \leq \frac{(1-\alpha) \delta(p, q)((j+p-q) !)}{\left(\frac{j}{p-q}+1\right)^{n}\left(\left(\frac{j}{p-q}(1-\alpha \lambda)\right)+(1-\alpha)\right)(j+p) !}
$$

So that

$$
\begin{aligned}
& \left|\frac{f(z)}{g(z)}-1\right|<\frac{\sum_{k=j+p}^{\infty}\left|a_{k}-b_{k}\right|}{1-\sum_{k=j+p}^{\infty} b_{k}} \\
& \leq \frac{\delta}{(j+p)}\left(\frac{1}{1-\frac{(1-\alpha) \delta(p, q)(j+p-q) !}{\left(\frac{j}{p-q}+1\right)^{n}\left(\left(\frac{j}{p-q}(1-\alpha \lambda)\right)+(1-\alpha)\right)(j+p) !}}\right)
\end{aligned}
$$

$$
\begin{aligned}
& =\frac{\delta(j+p) !\left(\frac{j}{p-q}+1\right)^{n}\left[\frac{j}{p-q}(1-\alpha \lambda)+(1-\alpha)\right]}{\left((j+p)\left(\frac{j}{p-q}+1\right)^{n}\left(\left(\frac{j}{p-q}(1-\alpha \lambda)\right)+(1-\alpha)\right)\right)(j+p) !-((1-\alpha) \delta(p, q))(j+p-q) !} \\
& =p-\gamma
\end{aligned}
$$

Provided that $\gamma$ is given properly by (16) .Thus we have $f(z) \in T_{j}(n, p, q, \alpha, \lambda, \gamma)$ for every $\gamma$ given by (16). This obviously completes the proof of Theorem (4).

5- Properties involving the operator $J_{c, p}$ and $D_{z}^{\mu}$ Lemma 1.[6] let the function $f(z) \in T(j, p)$, then $D_{z}^{\mu}\left(J_{c, p} f(z)\right)=$

$\frac{\Gamma(p+1)}{\Gamma(p-\mu+1)} z^{p-\mu}-\sum_{k=j+p}^{\infty}\left(\frac{(c+p) \Gamma(k+1)}{(c+k) \Gamma(k-\mu+1)}\right) a_{k} z^{k-\mu}$

$(\mu \in R ; c>-p ; j, p \in N)$

And

$$
\begin{aligned}
& J_{c, p}\left(D_{z}^{\mu} f(z)\right)=\frac{(c+p) \Gamma(p+1)}{(p-\mu+c) \Gamma(p-\mu+1)} z^{p-\mu}- \\
& \sum_{k=j+p}^{\infty}\left(\frac{(c+p) \Gamma(k+1)}{(k-\mu+c) \Gamma(k-\mu+1)}\right) a_{k} z^{k-\mu} \\
& (\mu \in R ; c>-p ; j, p \in N)
\end{aligned}
$$

Provided that there are no zeros appear in the denominators in (17) and (18). This in general, the operators

$J_{c, p}$ and $D_{z}^{\mu}$ are non-commutative .

So as to give growth and distortion properties for functions in the class $T_{j}(n, p, q, \alpha, \lambda)$ including the operators $J_{c, p}$ and $D_{z}^{\mu}$,we find it to be convenient to use the order operation exhibited by (18) and (19) as we shown in the following Theorems .

Theorem 5 .If $f(z)$ is in the class $T_{j}(n, p, q, \alpha, \lambda)$, then

$$
\begin{aligned}
& \left\{\frac{\Gamma(p+1)}{\Gamma(p-\mu+1)}-\right. \\
& \left.\left(\frac{(c+p) \Gamma(j+p+1)(1-\alpha) \delta(p, q)}{(j+p+c) \Gamma(j+p+\mu+1)\left(\frac{j}{p-q}+1\right)^{n}\left(\left(\frac{j}{p-q}(1-\alpha \lambda)\right)+(1-\alpha)\right)}\right)|z|^{j}\right\}|z|^{p+\mu} \leq \\
& \left|D_{z}^{-\mu}\left(J_{c, p} f(z)\right)\right| \\
& \leq \\
& \left\{\frac{\Gamma(p+1)}{\Gamma(p-\mu+1)}+\right. \\
& \left.\left(\frac{(c+p) \Gamma(j+p+1)(1-\alpha) \delta(p, q)}{(j+p+c) \Gamma(j+p+\mu+1)\left(\frac{j}{p-q}+1\right)^{n}\left(\left(\frac{j}{p-q}(1-\alpha \lambda)\right)+(1-\alpha)\right)}\right)|z|^{j}\right\}|z|^{p+\mu}
\end{aligned}
$$


Zainab .O/ Kassim .A

$(z \in U ; 0 \leq \alpha<p-q ; \mu>0 ; n, q \in$ $\left.N_{0} ; j, p \in N, c>-p ; p>q\right)$

(19)

The result is sharp for the function give by $J_{c, p}(f(z))=$

$Z^{p}-\left(\frac{(c+p)(1-\alpha) \delta(p, q)}{(j+p+c)\left(\frac{j}{p-q}+1\right)^{n}\left(\frac{j}{p-q}(1-\alpha \lambda)+(1-\alpha)\right)}\right) z^{j+p}$

(20)

Proof . It follows from Theorem (1) that

$$
\begin{aligned}
& \left(\frac{j}{p-q}+1\right)^{n}\left(\left(\frac{j}{p-q}(1-\alpha \lambda)\right)+(1-\alpha)\right) \delta(j+p, q) \sum_{k=j+p}^{\infty} a_{k} \leq \\
& \sum_{k=j+p}^{\infty}\left[\frac{k-q}{p-q}\right]^{n}\left(\left(\frac{k-q}{p-q}\right)-\alpha\left(1+\lambda\left(\left(\frac{k-q}{p-q}\right)-1\right)\right)\right) \delta(k, q) a_{k} \\
& \leq(1-\alpha) \delta(p, q) .
\end{aligned}
$$

Which readily yields

$\sum_{k=j+p}^{\infty} a_{k} \leq$

$\frac{(1-\alpha) \delta(p, q)}{\left(\frac{j}{p-q}+1\right)^{n}\left(\left(\frac{j}{p-q}(1-\alpha \lambda)\right)+(1-\alpha)\right) \delta(j+p, q)}$

(21)

Assumed that the function defined in $U$ by

$$
\begin{aligned}
& F(z)=\left(\frac{\Gamma(p+\mu+1)}{\Gamma(p+1)}\right) z^{-\mu} D_{z}^{-\mu}\left(J_{c, p} f(z)\right) \\
& =z^{p}-\sum_{k=j+p}^{\infty}\left(\frac{(p+c) \Gamma(p+\mu+1) \Gamma(k+1)}{(\mathrm{k}+\mathrm{c}) \Gamma(p+1) \Gamma(k+\mu+1)}\right) a_{k} z^{k} \\
& =\delta(p, q) z^{p-q}-\sum_{k=j+p}^{\infty} \theta(k) a_{k} z^{k-q} \quad(z \in U),
\end{aligned}
$$

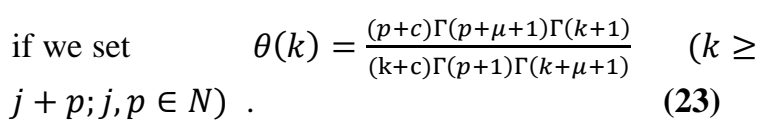

Then it is easily seen that $\theta(k)$ is decreasing function of $k$ when $\mu>0$,and hence

$\frac{(p+c) \Gamma(p+\mu+1) \Gamma(j+p+1)}{(\mathrm{j}+\mathrm{p}+\mathrm{c}) \Gamma(p+1) \Gamma(j+p+\mu+1)}$

$$
0<\theta(k) \leq \theta(j+p)=
$$

N)) . (24)

Where

$$
(c>-p: \mu>0 ; j, p \in
$$

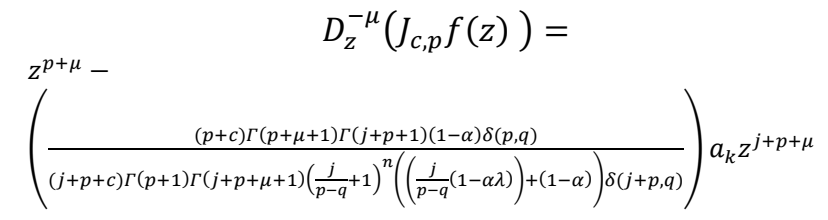

By using (21) and (24), we deduce that $|z|^{p}-\theta(j+p)|z|^{j+p} \sum_{k=j+p}^{\infty} a_{k} \leq|F(z)| \leq$

$$
|z|^{p}+\theta(j+p)|z|^{j+p} \sum_{k=j+p}^{\infty} a_{k}
$$

That is

$|z|^{p}-$

$\left(\frac{(p+c) \Gamma(p+\mu+1) \Gamma(j+p+1)(1-\alpha) \delta(p, q)}{(j+p+c) \Gamma(p+1) \Gamma(j+p+\mu+1)\left(\frac{j}{p-q}+1\right)^{n}\left(\left(\frac{j}{p-q}(1-\alpha \lambda)\right)+(1-\alpha)\right) \delta(j+p, q)}\right)|z|^{j+p} \leq$

$|F(z)| \leq$

$\left(\frac{|z|^{p}+}{(j+p+c) \Gamma(p+1) \Gamma(j+p+\mu+1)\left(\frac{j}{p-q}+1\right)^{n}\left(\left(\frac{j}{p-q}(1-\alpha \lambda)\right)+(1-\alpha)\right) \delta(j+p, q)}\right)|z|^{j+p}$

Which yields inequality (19)

Theorem 6 .If $f(z)$ is in $T_{j}(n, p, q, \alpha, \lambda)$, then

$$
\begin{aligned}
& \left\{\frac{\Gamma(p+1)}{\Gamma(p-\mu+1)}-\right. \\
& \left.\left(\frac{(c+p) \Gamma(j+p+1)(1-\alpha) \delta(p, q)}{(j+p+c) \Gamma(j+p-\mu+1)\left(\frac{j}{p-q}+1\right)^{n}\left(\left(\frac{j}{p-q}(1-\alpha \lambda)\right)+(1-\alpha)\right)}\right)|z|^{j}\right\}|z|^{p-\mu} \leq \\
& \left|D_{z}^{\mu}\left(J_{c, p} f^{q}(z)\right)\right| \\
& \leq \\
& \left\{\begin{array}{l}
\frac{\Gamma(p+1)}{\Gamma(p-\mu+1)}+ \\
\left.\left(\frac{(c+p) \Gamma(j+p+1)(1-\alpha) \delta(p, q)}{(j+p+c) \Gamma(j+p+\mu+1)\left(\frac{j}{p-q}+1\right)^{n}\left(\left(\frac{j}{p-q}(1-\alpha \lambda)\right)+(1-\alpha)\right)}\right)|z|^{j}\right\}|z|^{p-\mu}
\end{array}\right. \\
& \left(z \in U ; 0 \leq \alpha<p-q ; 0 \leq \mu \leq 1 ; n, q \in N_{0} ; j, p \in\right. \\
& N, c>-p ; p>q) . \\
& (\mathbf{2 5})
\end{aligned}
$$

The result is sharp for the function give by (20) .

Proof . It follows from Theorem (1) that

$\sum_{k=j+p}^{\infty} k a_{k} \leq$

$$
\begin{aligned}
& \frac{(j+p)(1-\alpha) \delta(p, q)}{\left(\frac{j}{p-q}+1\right)^{n}\left(\left(\frac{j}{p-q}(1-\alpha \lambda)\right)+(1-\alpha)\right) \delta(j+p, q)} \quad(0 \leq \alpha< \\
& p: 0 \leq \mu \leq 1 ; j, p \in N), \quad \text { (26) }
\end{aligned}
$$

suppose that the function defined in $U$ as follows

$$
\begin{aligned}
& G(z)=\frac{\Gamma(p-\mu+1)}{\Gamma(p+1)} z^{\mu} D_{z}^{\mu}\left(J_{c, p} f(z)\right) \\
= & z^{p}-\sum_{k=j+p}^{\infty}\left(\frac{(p+c) \Gamma(p-\mu+1) \Gamma(k)}{(k+c) \Gamma(p+1) \Gamma(k-\mu+1)}\right) k a_{k} z^{k} \\
= & z^{p}-\sum_{k=j+p}^{\infty} \vartheta(k) k a_{k} z^{k} \quad(z \in U)
\end{aligned}
$$

if we set $\quad \vartheta(k)=\frac{(p+c) \Gamma(p-\mu+1) \Gamma(k)}{(k+c) \Gamma(p+1) \Gamma(k-\mu+1)} \quad(k \geq$ $j+p ; 0 \leq \mu<1 ; j, p \in N)$.

Then it is easily seen that $\vartheta(k)$ is decreasing function of $k$ when $\mu<1$, and hence 
$0<\vartheta(k) \leq \vartheta(j+p)=\frac{(p+c) \Gamma(p-\mu+1) \Gamma(j+p)}{(j+p+c) \Gamma(p+1) \Gamma(j+p-\mu+1)}$

$(c>-p ; p, j \in N ; 0 \leq \mu<1)$.

By using (26) and (29), we deduce that

$$
\begin{gathered}
|z|^{p}-\vartheta(j+p)|z|^{j+p} \sum_{k=j+p}^{\infty} k a_{k} \leq|F(z)| \leq \\
|z|^{p}+\vartheta(j+p)|z|^{j+p} \sum_{k=j+p}^{\infty} k a_{k}
\end{gathered}
$$

That

$|z|^{p}-$

$\left(\frac{(c+p) \Gamma(p-\mu+1) \Gamma(j+p+1)(1-\alpha) \delta(p, q)}{(\mathrm{j}+\mathrm{p}+\mathrm{c}) \Gamma(p+1) \Gamma(j+p-\mu+1)\left(\frac{j}{p-q}+1\right)^{n}\left(\left(\frac{j}{p-q}(1-\alpha \lambda)\right)+(1-\alpha)\right)}\right)|z|^{j+p} \leq$

$|F(z)| \leq$

$|z|^{p}+$

$\left(\frac{(c+p) \Gamma(p-\mu+1) \Gamma(j+p+1)(1-\alpha) \delta(p, q)}{(\mathrm{j}+\mathrm{p}+\mathrm{c}) \Gamma(p+1) \Gamma(j+p-\mu+1)\left(\frac{j}{p-q}+1\right)^{n}\left(\left(\frac{j}{p-q}(1-\alpha \lambda)\right)+(1-\alpha)\right)}\right)|z|^{j+p}$

Which yields inequality (25) .

\section{References}

1 . G.S.Salagean ,H.M.Hossen and M.K.Aouf, On certain classes of $p$-valent functions with negative coefficients. II, Studia Univ. BabesBolyai, Vol.69,No. 1(2004),77-85 .

2 . H.M. Srivastava and M.K. Aouf ,Acertain fractional derivative operator and its applications to a new class of analytic and univalent functions with negative coefficients. I and II, J. Math. Anal. Appl. ,171(1992),1-13; ibid. 192(1995),973-688 .

3 . H.M. Srivastava and S.Owa (Eds) current Topics in analytic function Theory, world scientific publishing Company ,Singapore ,New Jersey ,London, and Hongkong , (1992) 234251 .

4 . H.M. Srivastava ,S.owa and S.K. Chatterjea , Anote on certain classes of starlike functions, Rend. Sem. Math. Univ. padova ,77(1987),115124 .
5. M.K. Aouf ,On certain subclasses of multivalent functions with negative coefficients defined by using differential operator, Bull. Instil. of Math. Academia Sinica, Vol.5 ,No.2(2010),181-200.

6 . M.P. Chen ,H. Irmak and H.M. Srivastava ,Some families of Multivalently analytic functions with negative coefficients, J.Math. Anal. Appl.214(1997),674-690.

7. O. Altintas,H. Irmak and H.M. Srivastava, neighbuorhoods for certain subclasses of multivalently analytic functions defined by using a differential operator ,Dep. Of Math. Baskont Univ. Turkey and Univ. of Victoria ,Canada , Computers and Mathematics with Applications 55(2008),331-338

8 . R.J. Libera , Some classes of regular univalent functions, Proc. Amer. Math. Soc.,16(1969),755758 .

9. R.Yamakawa,Certain subclasses of p-valently starlike functions with coefficients; in H.M. Srivastava and S.Owa (Eds) current Topics in analytic function Theory, world scientific publishing Company ,Singapore ,New Jersey ,London, and Hongkong ,(1992) 234251 .

10 .S.D. Bernardi ,Convex and star like univalent functions ,Trans . Amer. Math. Soc. ,135(1969),429-446.

11. S.Owa ,On certain classes of p- valent functions with negative coefficients ,Simon stevin, 59(1985), 385-402.

12.S. Owa , On the distortion Theorems. I, Kyung book Math. J. 18(1978), 55-59 .

13. S.Owa, The quasi-Hadamard Products of Certain analytic functions, in: H.M.Srivastava and S.Owa (Eds) current Topics in analytic theory, world scientific publishing company,Singapore,New Jersey,London, and Hong kong,(1992),pp.234-251. 


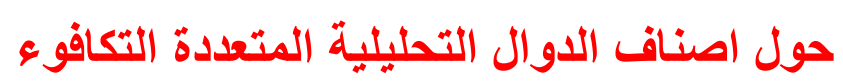

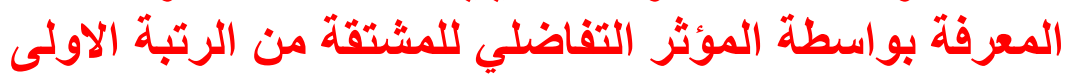

$$
\begin{aligned}
& \text { قاسم عبدالحميد جاسم } \\
& \text { قسم الرياضيات } \\
& \text { كلية العلوم } \\
& \text { جامعة بغداد } \\
& \text { قامتبة الرياضية عودة اثبينة }
\end{aligned}
$$

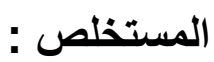

في البحث المقدم .وياستخدام العامل التفاضلي ، وجلنا حدود المعامل ، بعض الخواص المهمة لدوال التحليلية المتعددة التكافوء لمعاملات سالبه للصنف الجزئي(a)

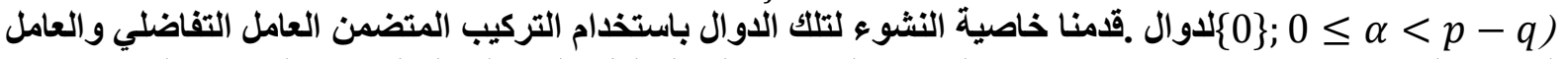
الكسري الحسابي مرة ومرة اخرى استخدمنا التركيب المتضمن العامل التفاضلي والعامل الكسري الحسابي المعكوس . 